Revista Colombiana de Obstetricia y Ginecología Vol. 63 No. 1 • Enero-Marzo 2012 • (46-56)

INVESTIGACIÓN ORIGINAL

\title{
PREVALENCIA DE RIESGO DE TRASTORNOS DE COMPORTAMIENTO ALIMENTARIO EN UNA POBLACIÓN FEMENINA DE ESTUDIANTES DE SECUNDARIA, MANIZALES, COLOMBIA, 2011
}

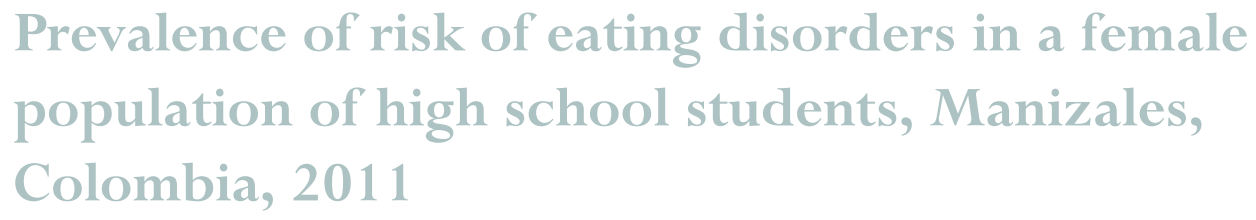

José Jaime Castaño Castrillón, Fis MSc*, Diana Lorena Giraldo**, Juliana Guevara**, Diana Lorena Losada **, Lina Marcela Meza *, Diana Melissa Narváez *, Liliana Carolina Sánchez ${ }^{* *}$, María Fernanda Sepúlveda**, Joana Velásquez-Chávez ${ }^{* *}$

Recibido: agosto 25/11 - Aceptado: marzo 6/12

\section{RESUMEN}

Objetivo: determinar la presencia del factor de riesgo (positivo o negativo) para trastornos de la conducta alimentaria (TCA) según el cuestionario EDI-2, y factores asociados en población de niñas escolarizadas de la ciudad de Manizales, Colombia.

Materiales y métodos: estudio de corte transversal en el que se aplicó la encuesta Eating Disorder Inventory (EDI-2) a estudiantes de tres colegios privados femeninos de la ciudad de Manizales. Criterios de inclusión: pertenecer a octavo, noveno, décimo y undécimo grado. Firma de consentimiento informado. Tamaño muestral: 481 estudiantes; muestreo de los colegios y los estudiantes: probabilístico estratificado por institución y grado. Variables medidas: sociodemográficas, Apgar familiar y medidas antropométricas; las subescalas contenidas en el EDI2 son: impulso a la delgadez, bulimia, insatisfacción corporal, inefectividad, perfeccionismo,

Profesor Titular. Director del Centro de Investigaciones, Facultad de Ciencias de la Salud, Universidad de Manizales, Caldas, Colombia. jcast@umanizales.edu.co.

** Estudiante X semestre del Programa de Medicina, Facultad de Ciencias de la Salud, Universidad de Manizales, Colombia. desconfianza interpersonal, conciencia interoceptiva, miedo a la madurez, ascetismo, regulación de impulsos e inseguridad social. El resultado se expresa como factor de riesgo positivo o negativo para trastornos de la conducta alimentaria. Es positivo si la subescala impulso a la delgadez tiene un valor mayor de 14. Análisis: medidas de tendencia central y dispersión para variables continuas, proporciones para variables categóricas.

Resultados: se encontró que el $24,7 \%$ de la población presentó factor de riesgo positivo según el cuestionario EDI-2 para desarrollar TCA, además se encontraron los siguientes factores asociados: consumo de alcohol $(p=0,002)$, antecedentes familiares de TCA ( $p=0,000)$, percepción de sobrepeso $(p=0,000)$, funcionalidad familiar $(0,000)$, índice de masa corporal ( $\mathrm{p}=0,032)$, y todas las medidas antropométricas excepto talla.

Conclusiones: la prevalencia de factor de riesgo para trastornos de la conducta alimentaria detectada en este estudio muestra que dichos trastornos siguen siendo un grave problema en las adolescentes colombianas. 
Palabras clave: trastornos de la conducta alimentaria, factores de riesgo, escolares, adolescente.

\section{ABSTRACT}

Objective: Determining the presence of eating behavior disorders (EBD) as a high risk factor (positive or negative) according to the EDI-2 questionnaire and associated factors in three schoolgirl populations in the city of Manizales, Colombia.

Materials and methods: this was cross-sectional study in which students from three private all-girls schools in the city of Manizales filled in the eating disorder inventory (EDI-2) questionnaire. The inclusion criteria involved being in the 8th, 9th, 10thor 11th grades (i.e. aged around 14-19). Informed consent forms were signed. . Sample size was 481 students; schools and students were probabilistically sampled and stratified by institution and grade. The variables measured were sociodemographic; family Apgar and anthropometric measurements were taken. The EDI-2 subscales used were: drive for thinness, bulimia, body dissatisfaction, ineffectiveness, perfectionism, interpersonal distrust, introspective awareness, fear of maturity, asceticism, regulation of impulsiveness and social insecurity. The result was expressed as a risk factor for positive or negative eating behavior disorders; this was positive if the drive for thinness subscale had a value greater than 14. Analysis involved measures of central tendency and dispersion for continuous variables and percentages for categorical variables.

Results: it was found that $24.7 \%$ of the study population had a positive risk factor for developing EBD according to the EDI-2 questionnaire. The following factors were found to be associated with an EBD: alcohol consumption ( $p=0.002)$, a family background of EBD $(p=0.000)$, perception of being overweight $(p=0.000)$, family functionality $(p=0.000)$, body mass index $(p=0.032)$ and practically all the anthropometric measurements, except size.

Conclusions: the prevalence of risk factors for eating behavior disorders detected in this study showed that EBD continues to be a serious problem in female colombian adolescents.

Key words: Eating behavior disorder, risk factor, female student, adolescent.

\section{INTRODUCCIÓN}

Los trastornos de la conducta alimentaria (TCA) son patologías complejas cuya etiopatogenia es multidimensional y responde a un modelo biopsicosocial, el riesgo de contraer una patología alimentaria surge de una combinación de variables (1), y una detección temprana de actitudes o conductas de riesgo puede prevenir el desarrollo de estos cuadros, mejorando la respuesta terapéutica y el pronóstico (2). Estos trastornos figuran entre los problemas de salud crónicos más frecuentes en los adolescentes, y su letalidad es la más alta entre los trastornos psiquiátricos (3). Practicar ejercicios o hacer dietas frecuentes constituyen factores de riesgo para TCA $(4,5)$. En la actualidad, la incidencia de TCA es cada vez más elevada y la población más afectada son las mujeres adolescentes (4-6) y la población menor de 25 años (7). En el estudio efectuado en España en el 2001 por Merino et al. se ha descrito además que los TCA se dan principalmente en personas jóvenes con un buen rendimiento intelectual, con éxito en sus estudios, son autoexigentes, perfeccionistas y desean agradar a los demás (8). Estos trastornos constituyen un problema que puede llegar a tener un alto impacto en la salud y calidad de vida, pueden llevar a la muerte o a consecuencias irreversibles $(9,10)$. En Colombia, los TCA cobran cada vez mayor importancia, la prevalencia de anorexia es de 0,55\%, para bulimia nerviosa de $1,2 \%$, y la frecuencia de trastornos no especificados es de 17,5\% en total 19,25\% (1,11-15). En particular, la población de niñas adolescentes manizaleñas está muy influenciada por sus pares de Medellín, en donde se sabe que el problema de los TCA es grave (12) debido a la tradición textilera y de alta costura de esta ciudad, que impulsa un modelo de belleza femenina de alta delgadez.

Las pacientes con TCA muestran mayor prevalencia de depresión, trastorno bipolar y abuso de 
sustancias (16). Son predictores de comportamientos de autoeliminación: el abuso sexual y físico en la infancia, la exposición a los medios de comunicación (17-19). Entre los TCA más frecuentes se encuentran la anorexia nerviosa y la bulimia nerviosa $(14,17,20)$; formas subclínicas como la preocupación excesiva por el peso, por las dietas, por la comida sana, y la insatisfacción y preocupación por la imagen corporal podrían tener tasas de prevalencia más elevadas que las formas clínicas (21). En el marco de los TCA la alteración de la imagen corporal tendría una participación causal (20-23).

Para medir los trastornos del comportamiento alimentario se ha utilizado el Eating Disorders Inventory 2 (EDI-2) (24), cuestionario de 91 preguntas, con las siguientes subescalas o dimensiones: impulso a la delgadez, bulimia, insatisfacción corporal, inefectividad, perfeccionismo, desconfianza interpersonal, conciencia interoceptiva, miedo a la madurez, ascetismo, regulación de impulsos e inseguridad social. Las tres primeras son relativas a los TCA, y las ocho restantes describen características psicológicas asociadas. El resultado del cuestionario se expresa como factor de riesgo (FR) positivo o negativo para trastornos de la conducta alimentaria. Es positivo si la subescala impulso a la delgadez tiene un valor mayor de 14. La confiabilidad y el constructo, así como la validez convergente y discriminante, han sido demostradas en repetidas ocasiones a través de su uso en población adolescente: ha sido validado en Chile en adolescentes escolarizadas de 13 a 18 años (25); en población análoga en México (26, 27); con adolescentes peruanas (28); en Colombia fue aplicado a población universitaria femenina y masculina (19), y en adolescentes escolarizadas (12); en mujeres mexicanas con diagnóstico de TCA (29); asimismo, fue validado en adolescentes tardías con diagnóstico de TCA (30). La versión del cuestionario empleada en este estudio fue adaptada para población colombiana por este grupo a partir de la versión argentina traducida y adaptada por Zukerfeld y validada por Melamed, Bunge et al. (citado por Restrepo) (12).
En vista de lo anterior, este estudio pretende determinar la presencia de factor de riesgo positivo para TCA según el cuestionario EDI-2, y hacer un análisis exploratorio de otros posibles factores de riesgo para TCA en población femenina de estudiantes de secundaria de la ciudad de Manizales, Colombia.

\section{MATERIALES Y MÉTODOS}

Estudio de corte transversal. Se estudiaron adolescentes de los grados $8^{\circ}, 9^{\circ}, 10^{\circ}$ y $11^{\circ}$ de tres colegios privados femeninos de la ciudad de Manizales, Colombia. Por solicitud expresa de las directivas del colegio 1 se tomó toda la población perteneciente a estos grados (254 estudiantes); para los colegios 2 y 3 se calculó el número de muestra con los siguientes parámetros: valor esperado 33\% (12) de factor de riesgo para TCA según EDI-2, margen de error 5\%, significancia 95\%. En estas condiciones, para el colegio 2 correspondió una muestra de 154 (población base 281) y para el colegio 3 la muestra fue de 63 estudiantes (población base 78) Para el cálculo del número de muestras se empleó la herramienta Statcalc del programa de libre distribución EPIINFO 3.3 (CDS). Esta muestra fue seleccionada mediante un muestreo probabilístico estratificado por institución y grado.

Aspectos éticos. Se solicitó el consentimiento informado de las directivas y de las estudiantes para definir la participación voluntaria, procedimiento que tuvo una duración de aproximadamente 30 min. Además, se guardó completa confidencialidad respecto a la identificación de estas, y se respetaron todas las disposiciones que plantearon las instituciones educativas implicadas.

Procedimiento. Previo al inicio del estudio se dio a conocer el proyecto respectivo en las instituciones participantes. Se realizó una prueba piloto en el segundo semestre del 2010 para comprobar la claridad del instrumento, el tiempo de respuesta y ajustar los programas de análisis estadístico. Para esta prueba se tomó el 5\% de la población del estudio. 
La prueba final se realizó en el primer semestre del 2011. Los integrantes del grupo de investigación aplicaron el instrumento asistiendo a las respectivas instituciones educativas durante el periodo académico de estas, además se hizo la recolección de las medidas antropométricas de cada estudiante.

Se evaluaron las siguientes variables: edad, peso corporal, talla, índice de masa corporal (IMC:peso/ talla 2), y se categorizaron en: bajo peso, peso adecuado, riesgo de sobrepeso, sobrepeso (31), perímetro de cintura, perímetro de cadera, índice cintura/cadera, perímetro abdominal, estrato socioeconómico, funcionalidad familiar medida con el Apgar familiar (categorizado como buena función, disfunción leve, disfunción moderada, disfunción severa) (32), consumo de alcohol, frecuencia de consumo (21) consumo de sustancias psicoactivas (tipo de sustancia psicoactiva), consumo de cigarrillo, frecuencia de consumo (semanal), actividad extracurricular (tipo de actividad), factor de riesgo para TCA medido con el cuestionario EDI-2, versión adaptada para Colombia: positivo, impulso a la delgadez $>14$; negativo, impulso a la delgadez $<=14$.

Análisis. Las variables de razón se describieron mediante promedios y desviaciones estándar, las variables nominales se describieron mediante tablas de frecuencia. Para determinar la relación entre variables nominales se empleó la prueba de $\chi^{2}$, y las de razón se determinaron mediante pruebas t, o análisis de varianza según el caso. Para medir la fiabilidad del cuestionario EDI-2 se calculó el alfa de Cronbach. Todos los análisis se efectuaron con una significancia $\alpha=0,05$. La base de datos se elaboró empleando el programa excel 2007 (Microsoft corporation), y los análisis estadísticos se realizaron con el programa IBM SPSS V.19 (IBM Corp.).

\section{RESULTADOS}

Se estudiaron un total de 481 niñas, de las cuales 254 provinieron del colegio 1, 154 del colegio 2, y 73 del colegio 3. La edad promedio fue de 14,26 años (DS $\pm 1,33)$, el 44,1\% de las niñas tenía buena función familiar (tabla1). El 1,7\% de los sujetos tenía antecedente de consumo de sustancias psicoactivas 7/ 401 (tabla 2). El cuestionario EDI-2 presentó un alfa de Cronbach de 0,802. Se encontró una frecuencia de antecedentes personales de TCA del 15,8\% según lo declarado por las estudiantes (considerados por ellas como antecedentes de TCA no diagnosticados); factor de riesgo positivo según EDI-2 para TCA, 24,7\% (IC 95\%: 21-28,9\%) de la población estudiada (tabla 3). Se encontró que de las estudiantes que consumen alcohol el 31,3\% (68/217) tienen factor positivo, mientras solo el 19,4\% (50/259) de las que no consumen alcohol tienen el factor de riesgo positivo (p: 0,002) para TCA según EDI-2. El 40,8\% (31/76) de las estudiantes que presentaban el antecedente familiar de TCA tenían factor positivo en comparación con solo el 21,9\% (87/398) de las que no tenían este antecedente familiar (P;0,0004).

Se encontró asociación significativa entre niñas con mayor IMC, mayores medidas antropométricas (excepto la talla) o con mala funcionalidad familiar, estas presentan una proporción mayor de FR positivo según EDI-2 para TCA y antecedente (tablas 4 y 5).

\section{DISCUSIÓN}

En la presente investigación se encontró un factor de riesgo positivo para TCA según EDI-2 de 24,7\% (IC95\%: 21-28,9\%) mayor al encontrado en la investigación realizada para población femenina en la universidad de Manizales en el año 2007 por Cano et al. (19), donde el factor de riesgo positivo fue del $17,3 \%$, y menor al compararlo con un estudio realizado en la ciudad de Medellín en el año 2003 por Restrepo (12), donde se encontró un factor positivo del 33\% en población similar a la del presente estudio. Fandiño et al. (15) reportan un 44,1\% de alta probabilidad de desarrollar algún TCA en población femenina de estudiantes de Medicina de la Universidad del Valle, empleando el cuestionario ECA. Rodríguez et al. (33), en la ciudad de Cali, Colombia, en el año 2005 realizaron un estudio en 198 niñas estudiantes de una institución educativa 


\begin{tabular}{|c|c|c|c|}
\hline Variable & Niveles & \# & $\%$ \\
\hline \multirow[t]{5}{*}{ Estrato social } & III & 202 & 42,1 \\
\hline & IV & 146 & 30,4 \\
\hline & VI & 59 & 12,3 \\
\hline & V & 51 & 10,6 \\
\hline & II & 22 & 4,6 \\
\hline \multirow[t]{4}{*}{ Grado } & 8 & 218 & 45,3 \\
\hline & 10 & 110 & 22,9 \\
\hline & 9 & 89 & 18,5 \\
\hline & 11 & 64 & 13,3 \\
\hline \multirow[t]{3}{*}{ Vive con } & Dos padres & 301 & 62,6 \\
\hline & Un padre & 155 & 32,2 \\
\hline & Otros familiares & 25 & 4,9 \\
\hline \multirow[t]{4}{*}{ Funcionalidad familiar } & Buena función & 211 & 44,1 \\
\hline & Disfunción leve & 159 & 33,2 \\
\hline & Disfunción moderada & 79 & 16,5 \\
\hline & Disfunción severa & 30 & 6,3 \\
\hline \multirow[t]{2}{*}{ Consumo de alcohol } & Sí & 218 & 45,4 \\
\hline & No & 262 & 54,6 \\
\hline \multirow[t]{4}{*}{ Frecuencia de consumo } & 1 & 74 & 34,6 \\
\hline & 2 & 67 & 31,3 \\
\hline & 3 & 26 & 12,1 \\
\hline & 4 & 15 & 7 \\
\hline \multirow[t]{4}{*}{ Tabaquismo según OMS } & No fuma & 446 & 93,5 \\
\hline & Fumador esporádico & 14 & 2,9 \\
\hline & Exfumador & 10 & 2,1 \\
\hline & Fumador habitual & 7 & 1,5 \\
\hline
\end{tabular}

(IEM); el muestreo fue no-probabilístico, y encontraron un riesgo para los TCA de 3,5\%, determinado con un cuestionario propio. Tanto en el estudio de Rodríguez como en el presente se reportó un 7,1\% de inducción de evacuaciones. Piñeros et al. (34), en su estudio realizado en 937 (59,6\% mujeres) estudiantes entre 12 y 20 años de edad de Bogotá y la Sabana de Bogotá, (Colombia, encontraron que entre la población femenina había un 27,2\% (119 de 437 niñas) de diagnóstico probable de TCA según el cuestionario EAT-26, valor muy cercano al 24,7\% encontrado en el presente estudio en población análoga. En el estudio en mención se realizó entrevista clínica estructurada para determinar TCA a 44 de las niñas con diagnóstico probable de TCA, de las cuales 28 (63,6\%) tuvieron este trastorno. Esta entrevista estructurada clínica también se realizó en el estudio de Restrepo et al. (12) a una muestra de niñas en riesgo y se encontró un 56\% de casos de TCA. En este estudio también se efectuó entrevista a una muestra que no presentaba FR para TCA positivo según EDI-2, encontrando un 3,6\% de casos de TCA (11).

Respecto a los estudios realizados en Iberoamérica, Aliaga-Deza et al. (28) -en una investigación publicada en el año 2010 y realizada en 39 adolescentes peruanos de ambos sexos, residentes en el Distrito Independencia de Lima, Perú- encontra- 


\begin{tabular}{|c|c|c|c|}
\hline & Niveles & $\#$ & $\%$ \\
\hline \multirow[t]{2}{*}{ TCA en la familia } & No & 401 & 83,9 \\
\hline & Sí & 77 & 16,1 \\
\hline \multirow[t]{7}{*}{ Cuál antecedente familiar de TCA } & Obesidad & 31 & 39,8 \\
\hline & Anorexia & 17 & 21,8 \\
\hline & Bulimia & 9 & 11,5 \\
\hline & Anorexia obesidad & 5 & 6,4 \\
\hline & Bulimia obesidad & 5 & 6,5 \\
\hline & Bulimia anorexia & 4 & 5,2 \\
\hline & Sobrepeso & 3 & 3,9 \\
\hline Antecedentes personales TCA & Sí & 76 & 15,8 \\
\hline \multirow[t]{12}{*}{ Cuáles } & Dieta frecuente & 28 & 36,8 \\
\hline & Sobrepeso & 20 & 20,3 \\
\hline & Obesidad & 1 & 1,3 \\
\hline & Anorexia & 5 & 6,5 \\
\hline & Bulimia & 4 & 5,3 \\
\hline & Desnutrición & 1 & 1,3 \\
\hline & Bajo peso & 1 & 1,3 \\
\hline & Sobrepeso dieta frecuente & 8 & 10,5 \\
\hline & Bulimia dieta frecuente & 5 & 6,5 \\
\hline & Anorexia bulimia & 1 & 1,3 \\
\hline & Anorexia bulimia-sobrepeso & 1 & 1,3 \\
\hline & Bulimia sobrepeso dieta frecuente & 1 & 1,3 \\
\hline \multirow[t]{5}{*}{ Inicio TCA (años) } & 12 & 25 & 37,3 \\
\hline & 13 & 14 & 20,9 \\
\hline & 10 & 9 & 13,5 \\
\hline & 11 & 5 & 7,5 \\
\hline & 15 & 2 & 3,0 \\
\hline \multirow[t]{2}{*}{ Actividades extracurriculares } & Sí & 283 & 59,3 \\
\hline & No & 193 & 40,7 \\
\hline \multirow[t]{4}{*}{ Cuál actividad } & Deporte & 131 & 46,8 \\
\hline & Danza & 40 & 14,2 \\
\hline & Otros & 26 & 10,3 \\
\hline & Danza deporte & 19 & 7,6 \\
\hline \multirow[t]{2}{*}{ Percepción de sobrepeso } & Sí & 215 & 44,8 \\
\hline & No & 265 & 55,2 \\
\hline \multirow[t]{2}{*}{ Come cuando siente ansiedad } & Sí & 183 & 38 \\
\hline & No & 298 & 62 \\
\hline \multirow[t]{2}{*}{ Induce evacuaciones } & Sí & 34 & 7,1 \\
\hline & No & 447 & 92,9 \\
\hline
\end{tabular}

ron un puntaje de 5,8 en la subescala EDI-2 de impulso a la delgadez, bastante menor al 9,6 obtenido en la presente investigación. En ese estudio no se calculó el factor de riesgo según EDI-2 para TCA, sin embargo, dado que esta subescala (impulso a la delgadez) es la que determina el factor de riesgo, 
Tabla 3.

Resultados de la aplicación del EDI2, y medidas antropométricas de adolescentes estudiantes de secundaria de tres colegios privados de la ciudad de Manizales, Caldas, 2011

\begin{tabular}{|c|c|c|c|c|c|}
\hline Variable & & $\#$ & & $\%$ & lc $95 \%$ \\
\hline \multirow[t]{2}{*}{ FR para TCA } & Positivo & 118 & & 24,7 & $21-28,9$ \\
\hline & Negativo & 359 & & 75,3 & $71,1-79$ \\
\hline \multirow[t]{4}{*}{ Clasificación del IMC } & Peso Adecuado & 388 & & 80,7 & $77,1-84,4$ \\
\hline & \multicolumn{2}{|l|}{ Riesgo Sobrepeso } & & 11,9 & $9,2-15,2$ \\
\hline & \multicolumn{2}{|l|}{ Bajo peso } & & 4,4 & $2,8-6,7$ \\
\hline & Sobrepeso & 13 & & 2,7 & $1,5-4,7$ \\
\hline \multicolumn{6}{|c|}{ Subescalas del EDI2 } \\
\hline \multicolumn{6}{|c|}{ LC 95\% } \\
\hline & P & $\mathrm{DE}$ & Rango & Li & Ls \\
\hline Impulso a la delgadez & 9,631 & 4,97 & 21 & 9,161 & 10,085 \\
\hline Bulimia & 6,167 & 3,79 & 20 & 5,820 & 6,515 \\
\hline Insatisfacción corporal & 9,878 & 5,30 & 27 & 9,401 & 10,356 \\
\hline Inefectividad & 8,305 & 5,49 & 17 & 8,026 & 8,583 \\
\hline Perfeccionismo & 8,543 & 4,05 & 18 & 8,165 & 8,92 \\
\hline Desconfianza Interpersonal & 8,086 & 5,49 & 27 & 7,592 & 8,58 \\
\hline Conciencia Introceptiva & 12,61 & 4,63 & 29 & 12,195 & 13,026 \\
\hline Miedo a la madurez & 12,32 & 3,40 & 22 & 0 & 22 \\
\hline Ascetismo & 10,363 & 3,46 & 22 & 10,053 & 10,674 \\
\hline Regulación de impulsos & 13,83 & 5,14 & 31 & 9,095 & 9,744 \\
\hline Inseguridad social & 9,42 & 3,62 & 22 & 9,095 & 9,744 \\
\hline \multicolumn{6}{|c|}{ Medidas antropométricas } \\
\hline Talla $(\mathrm{m})$ & 1,585 & 0,06 & 0,42 & 1,579 & 1,591 \\
\hline Peso $(\mathrm{kg})$ & 50,96 & 8,02 & 66,2 & 50,286 & 51,756 \\
\hline Perímetro cintura $(\mathrm{cm})$ & 67,85 & 6,42 & 41 & 67,25 & 68,44 \\
\hline Perímetro cadera $(\mathrm{cm})$ & 92,39 & 7,45 & 65 & 91,698 & 93,035 \\
\hline Perímetro abdominal & 79,87 & 8,53 & 52 & 79,08 & 80,61 \\
\hline Indice cintura/cadera & 0,736 & 0,062 & 0,69 & 0,73 & 0,742 \\
\hline $\mathrm{IMC}$ & 20,3 & 2,97 & 26,86 & 20,033 & 20,566 \\
\hline
\end{tabular}

es de esperarse que sea menor en la población peruana, puesto que si el promedio general es menor, con toda seguridad una menor proporción de niñas tienen un valor de 14 (punto de corte para FR positivo) o más en la subescala. En relación con las otras subescalas, los resultados presentados por esta población siempre fueron menores que los reportados por la población de niñas de la presente investigación.
De manera análoga al anterior estudio, Urzúa et al. (25), en su trabajo de validación del EDI-2 en adolescentes escolarizados chilenos, publicado en el año 2009, encontraron los siguientes valores en las niñas para las tres subescalas principales: 7,53; 2,19 y 8,85 , también menores a los reportados en el presente trabajo, de 9,6; 6,17 y 9,88. Lo mismo sucede con las otras subescalas. En este estudio tampoco se calcula el FR para TCA, pero dado que 


\begin{tabular}{|c|c|c|c|c|}
\hline & & $\begin{array}{c}\text { Pos } \\
(\mathrm{n}=118)\end{array}$ & $\begin{array}{c}\text { Neg } \\
(\mathrm{n}=359)\end{array}$ & p \\
\hline \multirow[t]{3}{*}{ Colegio } & 1 & $\begin{array}{c}71 \\
28 \%\end{array}$ & $\begin{array}{c}183 \\
72 \%\end{array}$ & 0,002 \\
\hline & 2 & $\begin{array}{c}23 \\
15,7 \%\end{array}$ & $\begin{array}{c}127 \\
84,7 \%\end{array}$ & \\
\hline & 3 & $\begin{array}{c}24 \\
32,91 \%\end{array}$ & $\begin{array}{c}49 \\
67,1 \%\end{array}$ & \\
\hline \multirow[t]{2}{*}{ Percepción de sobrepeso } & No & $\begin{array}{c}29 \\
11 \%\end{array}$ & $\begin{array}{c}234 \\
89,0 \%\end{array}$ & 0,000 \\
\hline & Sí & $\begin{array}{c}89 \\
41,8 \%\end{array}$ & $\begin{array}{c}124 \\
58,2 \%\end{array}$ & \\
\hline \multirow[t]{2}{*}{ Come cuando está ansiosa } & No & $\begin{array}{c}51 \\
17,2 \%\end{array}$ & $\begin{array}{c}245 \\
82,8 \%\end{array}$ & 0,000 \\
\hline & Sí & $\begin{array}{c}67 \\
37,0 \%\end{array}$ & $\begin{array}{c}114 \\
63,0 \%\end{array}$ & \\
\hline \multirow[t]{2}{*}{ Se induce evacuaciones } & No & $\begin{array}{c}96 \\
21,7 \%\end{array}$ & $\begin{array}{c}347 \\
78,3 \%\end{array}$ & 0,000 \\
\hline & Sí & $\begin{array}{c}22 \\
64,7 \%\end{array}$ & $\begin{array}{c}12 \\
35,3 \%\end{array}$ & \\
\hline \multirow[t]{4}{*}{ Funcionalidad familiar } & Buena función & $\begin{array}{c}40 \\
19,2 \%\end{array}$ & $\begin{array}{c}168 \\
80,8 \%\end{array}$ & 0,000 \\
\hline & Disfunción leve & $\begin{array}{c}32 \\
20,3 \%\end{array}$ & $\begin{array}{c}126 \\
79,7 \%\end{array}$ & \\
\hline & Disfunción moderada & $\begin{array}{c}35 \\
44,3 \%\end{array}$ & $\begin{array}{c}44 \\
55,7 \%\end{array}$ & \\
\hline & Disfunción severa & $\begin{array}{c}11 \\
36,7 \%\end{array}$ & $\begin{array}{c}19 \\
63,3 \%\end{array}$ & \\
\hline
\end{tabular}

la escala que lo determina tiene valor promedio menor, debe ser igualmente menor.

En otro estudio, realizado por González-Juárez et al. (35) en Madrid, España, en el año 2007, aplicado a una población de 8754 jóvenes entre los 12 y 18 años de ambos sexos, pertenecientes a colegios públicos de Leganés (Madrid), se encontró que el $11,8 \%$ de las niñas están en riesgo de presentar un TCA, valor bastante menor al encontrado en el presente trabajo, aunque determinado con otro cuestionario, el EAT-26. En este estudio también se encontró relación significativa entre el FR para TCA e inducirse evacuaciones.

Respecto a los factores de riesgo, en una investigación hecha por Casal et al. (36), también en Madrid, en el año 2011, donde se analizaron 101 historias clínicas de pacientes con TCA, se encontró una relación significativa entre consumo de alcohol y sustancias psicoactivas y el desarrollo de factores asociados para TCA con un porcentaje de 86,9\%. Hernández et al. (37) realizaron un estudio en la ciudad de México en el año 2010 planteado 


\begin{tabular}{|c|c|c|c|c|c|}
\hline \multirow[t]{2}{*}{ Variable } & \multicolumn{2}{|c|}{ Positivo $=118$} & \multicolumn{2}{|c|}{ Negativo $=359$} & \multirow[b]{2}{*}{$\mathbf{p}$} \\
\hline & $\mathbf{P}$ & $\mathrm{DE}$ & $\mathbf{P}$ & DE & \\
\hline Puntaje Apgar & 14,69 & 4,53 & 16,3 & 3,69 & 0,000 \\
\hline Peso (kg) & 53,6 & 9,2 & 50,1 & 7,4 & 0,000 \\
\hline Perímetro cintura (cm) & 69,24 & 6,39 & 67,36 & 6,3 & 0,005 \\
\hline Perímetro cadera (cm) & 94.17 & 8,18 & 91.78 & 7,08 & 0,002 \\
\hline Perímetro abdomen (cm) & 82,22 & 8,81 & 79,12 & 8,28 & 0,001 \\
\hline Pliegue en brazo & 14,94 & 4,34 & 13,37 & 3,73 & 0,000 \\
\hline IMC (numérico) & 21,41 & 3,28 & 19,91 & 2,74 & 0,000 \\
\hline \multirow[t]{5}{*}{$\begin{array}{l}\text { Categorías de índice de } \\
\text { masa corporal }\end{array}$} & Bajo peso & $\begin{array}{c}2 \\
9,5 \%\end{array}$ & \multicolumn{2}{|l|}{$\begin{array}{c}19 \\
90,5 \%\end{array}$} & \\
\hline & Peso adecuado & $\begin{array}{c}93 \\
24,1 \%\end{array}$ & \multicolumn{2}{|l|}{$\begin{array}{c}293 \\
75,9 \%\end{array}$} & \\
\hline & Riesgo sobrepeso & $\begin{array}{c}15 \\
27,3 \%\end{array}$ & $\begin{array}{c}40 \\
72,7 \%\end{array}$ & & \\
\hline & Sobrepeso & $\begin{array}{c}7 \\
53,8 \%\end{array}$ & \multicolumn{2}{|l|}{$\begin{array}{c}6 \\
46,2 \%\end{array}$} & \\
\hline & \multicolumn{4}{|c|}{ Factor de riesgo para TCA según EDI2 } & \\
\hline
\end{tabular}

en una población femenina mexicana $(\mathrm{n}=44)$ con diagnóstico de TCA, se encontró que el 40\% presentaban un antecedente de TCA en la familia, comparado con el resultado de 16,1\% obtenido en la presente investigación. Al igual que en el estudio de Cruzart et al. (3), en este trabajo también se encontró relación significativa entre el FR para TCA según EDI-2, y funcionalidad familiar.

Los resultados reportados en el presente estudio, junto con los datos encontrados en otras investigaciones efectuadas en poblaciones de niñas escolarizadas de Colombia, muestran que una de cada cuatro niñas presenta FR para TCA, y confirman que este sigue siendo un grave problema para las adolescentes colombianas. Según lo reportado en estudios efectuados en Chile y Perú (3, 25), el FR para TCA en niñas de estos países es menor que el presentado por las niñas colombianas.

Durante el desarrollo de la investigación se pre- sentaron varias limitaciones, tales como la falta de colaboración por parte de algunas niñas, pues no todas permitían la toma de medidas antropométricas generando falta de información en cuanto a este aspecto, por lo cual se excluyeron del presente estudio. La limitación del tiempo para contestar el cuestionario constituyó otra dificultad, pues algunas de las niñas manifestaron requerir mayor tiempo para hacerlo, hecho que puede influir en la exactitud de sus respuestas.

\section{CONCLUSIÓN}

Los resultados del presente estudio muestran una frecuencia de factor de riesgo para TCA de una de cada cuatro niñas, resultados que coinciden con los de otros estudios realizados en Colombia, y que además muestran que este problema no es solo de las niñas "paisas". Lo anterior indica que el riesgo de adquirir TCA en las niñas colombianas continúa 
siendo alto. Se concluye que los efectos del estereotipo de belleza siguen siendo devastadores en las niñas colombianas, y al parecer no se llevan a cabo campañas que pongan este estereotipo en su justo sitio.

\section{REFERENCIAS}

1. Castro-Betancourt M. Trastornos de la conducta alimentaria: los gramos que pesan en la mente. Bogotá: Pontificia Universidad Javeriana, Trabajo de grado para optar por el título de Comunicadora social y Periodista; 2008.

2. Behar R, Alviña M, González T, Rivera N. Detección de actitudes y/o conductas predisponentes a trastornos alimentarios en estudiantes de enseñanza media de tres colegios particulares de Viña del Mar. Rev Chil Nutr 2007;34:240-9.

3. Cruzart C, Ramírez P, Melipillan R, Marzolo P. Trastornos alimentarios y funcionamiento familiar percibido en una muestra de estudiantes de secundaria de la comuna de Concepción, Chile. Psykhe 2008;17:81-90.

4. Behar R. La alteración de la imagen corporal en los trastornos de la conducta alimentaria: aspectos biopsicosociales. RNC 2008;18:109-17.

5. Patton GC, Selzer R, Coffey C, Carlin JB, Wolfe R. Onset of adolescent eating disorders: population based cohort study over 3 years. BMJ 1999;318:765-8.

6. Acosta V, Llopis JM, Gómez G, Pineda G. Evaluación de la conducta alimentaria de riesgo. Estudio transcultural entre adolescentes de España y México. International Journal Psychology and Psychological Therapy 2005;5:223-32.

7. Suárez F, Vaz F, Guisado JA, Gómez L. Estudio de hábitos alimentarios en población femenina comparando grupos con patología y sin patología alimentaria. Nutr Hosp 2003;18:259-63.

8. Merino H, Pombo MG, Godás A. Evaluación de actitudes alimentarias y la satisfacción corporal en una muestra adolescente. Psicothema 2001; 13:539-45.

9. Ángel LA, Martínez LM, Gómez MT. Prevalencia de trastornos del comportamiento alimentario (TCA) en estudiantes de bachillerato. Rev Fac Med Unal 2008;56:193-210.
10. Bay LB, Raush C, Kovalskys I, Berner E, Orellana L, Bergesio A. Alteraciones alimentarias en niños y adolescentes argentinos que concurren al consultorio del pediatra. Arch Argent Pediatr 2005;103: 305-16.

11. Rueda GE, Díaz LA, Pinzón C, Rodríguez J, Cadena L. Validación del cuestionario SCOFF para el cribado de los trastornos del comportamiento alimentario en adolescentes escolarizadas. Aten Primaria 2005;35:89-94.

12. Restrepo A, Vélez S, Isaza M, Isaza A, Aristizábal G, Palacio L. Prevalencia de los trastornos de la conducta alimentaria en mujeres estudiantes de secundaria de la ciudad de Medellín y el área metropolitana. Departamento de Psiquiatría, Área Salud Mental de las Mujeres, Programa Trastornos de la Conducta Alimentaria, Facultad de Medicina Servicio Seccional de Salud de Antioquia; 2003.

13. Rueda G, Cadena A, Díaz LF, Ortiz DP, Pinzón C, Rodríguez J. Validación de la encuesta de comportamiento alimentario en adolescentes escolarizadas de Bucaramanga, Colombia. Rev Colomb Psiquiatr 2005;34:375-85.

14. Castrillón D, Luna I, Avendaño G, Pérez AM. Validación del body shape questionaire BSQ para la población colombiana. Acta Colom Psicol 2007;10:15-23.

15. Fandiño A, Giraldo S, Martínez C, Aux C, Espinosa R. Factores asociados con los trastornos de la conducta alimentaria en estudiantes universitarios en Cali, Colombia. Colomb Med 2007;38:344-51.

16. Rodríguez-Guarín M. Uso de sustancias, impulsividad y trauma en pacientes con trastornos del comportamiento alimentario: una tríada de riesgo para comorbilidades complejas que afectan el pronóstico. Rev Colomb Psiquiat 2009;38:420-32.

17. Rodríguez GM, Guerrero S. Frecuencia y fenomenología de lesiones autoinfligidas en mujeres colombianas con trastornos del comportamiento alimentario. Rev Colomb Psiquiatr 2005;34:343-54.

18. Taylor CB, Bryson S, Celio DA, Luce K, Cunning D, Abascal LB, et al. The adverse effect of negative comments about weight and shape from family and siblings on women at high risk for eating disorders. Pediatrics 2006;118:731-8. 
19. Cano A, Castaño J, Corredor DA, García AM, González M, Lloreda OL, et al. Factores de riesgo para trastornos de la alimentación en los alumnos de la universidad de Manizales. MedUNAB 2007;10:187-94.

20. Yager J, Andresen AE. Clinical practice. Anorexia Nervosa. N Engl J Med 2005;353:1481-8.

21. Ballester D, De Gracia M, Patiño J, Suñol C, Ferrer M. Actitudes alimentarias y satisfacción corporal en adolescentes: un estudio de prevalencia. Actas Esp Psiquiatr 2002;30:207-12.

22. Lameiras M, Calado M, Rodríguez Y, Fernández M. Hábitos alimentarios e imagen corporal en estudiantes universitarios sin trastornos alimenticios. RIPCS/ IJCHP 2003;3:23-33.

23. Espina A, Ortego MA, Ochoa I, Yenes F, Aleman A. La imagen corporal en los trastornos alimentarios. Psicothema 2001;13:533-8.

24. Garner D. EDI-2, Eating Disorder Inventory-2, Professional Manual. Odessa: PAR, Psychological Assessment Resources, Inc.; 1990.

25. Urzúa A, Castro S, Lillo A, Leal C. Evaluación de los trastornos alimentarios: propiedades psicométricas del test EDI-2 en adolescentes escolarizados(as) de 13 a 18 años. Rev Chil Nutr 2009;36:1063-73.

26. Ochoa-Alcaraz SG. Validación y confiabilidad del inventario de trastornos de la conducta alimentaria (edi-2) aplicado en una muestra de adolescentes mexicanos. Revista Científica Electrónica de Psicología Icsa-UAEH 2008; 5:30-40.

27. García-García E, Vásquez-Velázquez V, López-Alvarenga J, Arcila-Martínez D. Validez interna y utilidad diagnóstica del Eating Disorders Inventory en mujeres mexicanas. Salud Publica Mex 2003;45:206-10.

28. Aliaga-Deza L, De La Cruz-Saldaña TA, VegaDienstmaier JM. Sintomatología de los trastornos de la conducta alimentaria en adolescentes de un colegio del distrito de Independencia, Lima, Perú. Rev Neuro Psiquiatr 2010;73:52-61.
29. Unikel-Santoncini C, Bojorquez-Chapela S, CarreñoGarcía S, Caballero-Romo A. Validación del Eating Disorder Inventory en una muestra de mujeres mexicanas con trastorno de la conducta alimentaria. Salud Mental 2006;29:44-51.

30. Quiroga S, Cryan G. Resultados del Inventario EDIII en Adolescentes Tardías Femeninas con Trastornos de la Alimentación. Comparación de Tratamientos. Fundamentos en Humanidades, Universidad Nacional de San Luis, Argentina 2007;7:127-52.

31. Organización Mundial de la Salud. Patrones de crecimiento del Niño de la OMS. Ginebra: OMS; 2006.

32. Forero LM, Avendaño MC, Duarte ZJ, Campo A. Consistencia interna y análisis de factores de la escala Apgar para evaluar el funcionamiento familiar en estudiantes de básica secundaria. Rev Colomb Psiquiatr 2006;35:23-9.

33. Rodríguez JM, Mina FJ. Prevalencia de factores de riesgo asociados a trastornos del comportamiento alimentario en adolescentes de una institución educativa en Cali, Colombia 2005. Rev Colomb Obstet Ginecol; 59:180-9.

34. Piñeros S, Molano J, López C. Factores de riesgo de los trastornos de la conducta alimentaria en jóvenes escolarizados en Cundinamarca, Colombia. Rev Colomb Psiquiatr 2010;39:313-28.

35. González-Juárez C, Pérez-Pérez E, Martín-Cabrera B, Mitja-Pau I, Roy de Pablo R, Vásquez de la Torre P. Detección de adolescentes en riesgo de presentar trastornos de la alimentación. Aten Primaria 2007;39:189-94.

36. Casal P, Maldonado MJ, Ferre F. Estudio de los perfiles clínicos de los pacientes con trastorno de la conducta alimentaria en dispositivos específicos. Actas Esp Psiquatr 2011;39:12-9.

37. Hernández RC, Aizpuru de la Portilla A. Antecedentes familiares y sintomatología en mujeres con trastornos de conducta alimentaria. Revista Mexicana de Trastornos Alimentarios 2010;1:119-24. 